\title{
Association of a polymorphism of BTN2A1 with chronic kidney disease in community-dwelling individuals
}

\author{
MITSUTOSHI OGURI ${ }^{1}$, TETSUO FUJIMAKI ${ }^{2}$, HIDEKI HORIBE ${ }^{3}$, KIMIHIKO KATO $^{4,5}$, \\ SAHOKO ICHIHARA $^{5}$ and YOSHIJI YAMADA ${ }^{5}$
}

\author{
${ }^{1}$ Department of Cardiology, Japanese Red Cross Nagoya First Hospital, Nagoya, Aichi; ${ }^{2}$ Department of Cardiovascular \\ Medicine, Inabe General Hospital, Inabe, Mie; ${ }^{3}$ Department of Cardiovascular Medicine, Gifu Prefectural \\ Tajimi Hospital, Tajimi, Gifu; ${ }^{4}$ Meitoh Hospital, Nagoya, Aichi; ${ }^{5}$ Department of Human Functional \\ Genomics, Life Science Research Center, Mie University, Tsu, Mie, Japan
}

Received September 06, 2013; Accepted September 23, 2013

DOI: $10.3892 /$ br.2013.176

\begin{abstract}
Results of recent studies have shown that the $\mathrm{C} \rightarrow \mathrm{T}$ polymorphism (rs6929846) of the butyrophilin, subfamily 2 , member A1 gene (BTN2Al) was significantly associated with myocardial infarction. The aim of the current study was to examine the association of rs6929846 of BTN2Al with chronic kidney disease (CKD) in community-dwelling individuals. Study subjects comprised 1,709 community-dwelling individuals, including 435 subjects with CKD [estimated glomerular filtration rate $(\mathrm{eGFR})<60 \mathrm{ml} / \mathrm{min}$ per $\left.1.73 \mathrm{~m}^{2}\right]$ and 1,274 controls $\left(\mathrm{eGFR} \geq 90 \mathrm{ml} / \mathrm{min}\right.$ per $1.73 \mathrm{~m}^{2}$ ) who were recruited to a population-based cohort study. Genotype distributions $(\mathrm{P}=0.0010)$ and allele frequencies $(\mathrm{P}=0.0002)$ of rs6929846 were significantly associated with CKD. Multivariate logistic regression analysis with adjustment for covariates revealed that the rs6929846 of BTN2A1 was significantly ( $\mathrm{P}=0.0002$; odds ratio, 2.02 ; dominant model) associated with $\mathrm{CKD}$, with the minor $\mathrm{T}$ allele representing a risk for this condition. The serum concentrations of creatinine were significantly $(\mathrm{P}=0.0107)$ higher for all the individuals, whereas eGFR was significantly $(\mathrm{P}=0.0468)$ lower for individuals in the combined group of CT and TT genotypes compared to those with the CC genotype. BTN2A1 may therefore be a susceptibility gene for CKD.
\end{abstract}

\section{Introduction}

Chronic kidney disease (CKD) is a growing public health concern worldwide, considering that individuals with CKD are at increased risk for end-stage renal disease as well as for cardiovascular diseases and premature death (1-3). For the purpose of improvement of prognosis and cost-effectiveness,

Correspondence to: Dr Yoshiji Yamada, Department of Human Functional Genomics, Life Science Research Center, Mie University, 1577 Kurima-machiya, Tsu, Mie 514-8507, Japan

E-mail: yamada@gene.mie-u.ac.jp

Key words: genetics, genetic epidemiology, polymorphism, chronic kidney diseases, end-stage renal disease aggressive prevention and early detection is imperative as a global approach to CKD (4).

In Japan, a nationwide health screening program was begun in order to control lifestyle-related diseases, particularly metabolic syndrome in 2008 (5). Results of previous studies showed that use of dipstick urinalysis to detect proteinuria but was inadequate in the routine screening for the detection of early stage CKD due to insufficient sensitivity and specificity (6). In the general population, screening combined with serum concentrations of creatinine or other biomarkers is therefore likely to be appropriate and cost-effective for the stratification of high-risk individuals (7). Identification of genetic variants that confer susceptibility to CKD may thus be important in determining which patients are recommended for frequent checkup.

Mounting evidence indicates that hypertension, diabetes mellitus, and dyslipidemia are major risk factors for the development and progression of CKD (8-11). In addition to these conventional risk factors, genetic epidemiological studies have demonstrated that genetic factors are key in the development of CKD in the general population $(12,13)$. Although recent genome-wide association studies (GWASs) identified various loci and genes indicating a predisposition to CKD $(14,15)$, the genes that contribute to genetic susceptibility to CKD in Japanese individuals remain to be identified definitively.

In a recent study, we showed that the $\mathrm{C} \rightarrow \mathrm{T}$ polymorphism (rs6929846) in the butyrophilin, subfamily 2, member A1 gene (BTN2A1) was significantly associated with myocardial infarction in Japanese individuals by a GWAS (16). Since CKD is considered an important risk factor for coronary heart disease, we hypothesized that the association of rs6929846 of BTN2Al with myocardial infarction might be partially attributable to its effects on susceptibility to CKD. Therefore, an association study for rs6929846 of BTN2A1 and CKD was performed in community-dwelling Japanese individuals to provide a basis for the personalized prevention of this condition.

\section{Materials and methods}

Study population. The study population comprising 1,709 community-dwelling Japanese individuals (435 subjects 
Table I. Characteristics of study subjects.

\begin{tabular}{|c|c|c|c|}
\hline Characteristics & CKD & Controls & P-value \\
\hline No. of subjects & 435 & 1,274 & \\
\hline Age (years) & $65.5 \pm 9.6$ & $46.4 \pm 11.5$ & $<0.0001$ \\
\hline Gender (male/female, \%) & $66.2 / 33.8$ & $49.1 / 50.9$ & $<0.0001$ \\
\hline Body mass index $\left(\mathrm{kg} / \mathrm{m}^{2}\right)$ & $23.4 \pm 3.1$ & $22.7 \pm 3.7$ & $<0.0001$ \\
\hline Current or former smoker $(\%)$ & 32.9 & 41.4 & 0.0017 \\
\hline Hypertension (\%) & 57.5 & 19.2 & $<0.0001$ \\
\hline Systolic blood pressure (mmHg) & $125 \pm 17$ & $117 \pm 15$ & $<0.0001$ \\
\hline Diastolic blood pressure (mmHg) & $76 \pm 12$ & $72 \pm 12$ & $<0.0001$ \\
\hline Dyslipidemia (\%) & 61.2 & 39.1 & $<0.0001$ \\
\hline Serum total cholesterol (mmol/l) & $5.16 \pm 0.91$ & $5.02 \pm 0.84$ & 0.0475 \\
\hline Serum triglycerides $(\mathrm{mmol} / \mathrm{l})$ & $1.38 \pm 0.81$ & $1.20 \pm 0.99$ & $<0.0001$ \\
\hline Serum HDL-cholesterol (mmol/l) & $1.54 \pm 0.43$ & $1.68 \pm 0.45$ & $<0.0001$ \\
\hline Serum LDL-cholesterol (mmol/l) & $3.17 \pm 0.79$ & $3.08 \pm 0.83$ & 0.0157 \\
\hline Diabetes mellitus (\%) & 16.6 & 8.7 & $<0.0001$ \\
\hline Fasting plasma glucose (mmol/l) & $5.69 \pm 1.03$ & $5.58 \pm 1.52$ & $<0.0001$ \\
\hline Blood glycosylated hemoglobin $(\%)$ & $5.9 \pm 0.6$ & $5.7 \pm 0.8$ & $<0.0001$ \\
\hline Blood urea nitrogen (mmol/l) & $6.32 \pm 1.81$ & $4.50 \pm 1.18$ & $<0.0001$ \\
\hline Serum creatinine $(\mu \mathrm{mol} / \mathrm{l})$ & $79.3 \pm 20.8$ & $45.3 \pm 7.9$ & $<0.0001$ \\
\hline eGFR (ml/min per $\left.1.73 \mathrm{~m}^{2}\right)$ & $52.8 \pm 7.5$ & $100.6 \pm 9.7$ & $<0.0001$ \\
\hline
\end{tabular}

Quantitative data are means \pm standard deviation. CKD, chronic kidney disease HDL, high-density lipoprotein; LDL, low-density lipoprotein; eGFR, estimated glomerular filtration rate.

with CKD and 1,274 controls) were recruited to a population-based cohort study in Inabe City (Mie Prefecture, Japan) during the period of March 2010 and September 2012.

Estimated glomerular filtration rate (eGFR) was calculated with the use of the simplified prediction equation derived from the modified version of that described in the Modification of Diet in Renal Disease (MDRD) study as proposed by the Japanese Society of Nephrology (17): eGFR $\left(\mathrm{ml} / \mathrm{min}\right.$ per $\left.1.73 \mathrm{~m}^{2}\right)=194 \mathrm{x}$ [age $($ years $\left.)\right]^{-0.287} \mathrm{x}$ [serum creatinine $(\mathrm{mg} / \mathrm{dl})]^{-1.094} \times$ [0.739 if female]. The National Kidney Foundation-Kidney Disease Outcomes Quality Initiative guidelines recommend a diagnosis of CKD if eGFR is $<60 \mathrm{ml} / \mathrm{min}$ per $1.73 \mathrm{~m}^{2}$ (18). On the basis of this criterion, 435 subjects (288 males and 147 females) were diagnosed with CKD. The control subjects comprised 1,274 individuals (625 males and 649 females) whose eGFR was $\geq 90 \mathrm{ml} / \mathrm{min}$ per $1.73 \mathrm{~m}^{2}$. Control individuals had no renal disease or major health problems. Subjects with CKD and controls had or did not have conventional risk factors for CKD, including hypertension (systolic blood pressure of $\geq 140 \mathrm{mmHg}$, diastolic blood pressure of $\geq 90 \mathrm{mmHg}$, or taking antihypertensive medication), diabetes mellitus (fasting plasma glucose of $\geq 6.93 \mathrm{mmol} / 1$, blood hemoglobin A1c content of $\geq 6.9 \%$, or taking antidiabetes medication) or dyslipidemia (a serum concentration of triglycerides of $\geq 1.65 \mathrm{mmol} / 1$, a serum high-density lipoprotein (HDL)-cholesterol of $<1.04 \mathrm{mmol} / \mathrm{l}$, a serum low-density lipoprotein (LDL)-cholesterol of $\geq 3.64 \mathrm{mmol} / \mathrm{l}$ or had taken antidyslipidemic medication).

The study protocol complied with the Declaration of Helsinki and was approved by the ethics committees of Human
Research of Mie University Graduate School of Medicine and Inabe General Hospital. Written informed consent was obtained from each subject.

Genotyping of a polymorphism. Venous blood $(5 \mathrm{ml})$ was collected into tubes containing $50 \mathrm{mmol} / \mathrm{l}$ ethylenediaminetetraacetic acid (disodium salt), peripheral blood leukocytes were isolated, and genomic DNA was extracted from these cells using a DNA extraction kit (SMITEST EX-R\&D; Medical \& Biological Laboratories Co., Ltd., Nagoya, Japan). Genotypes of rs6929846 of BTN2A1 were determined at G\&G Science Co., Ltd. (Fukushima, Japan) by the multiplex bead-based Luminex assay, a method that combines polymerase chain reaction $(\mathrm{PCR})$ and sequence-specific oligonucleotide probes with suspension array technology (Luminex, Austin, TX, USA). Genotyping involved PCR amplification, hybridization, streptavidin-phycoerythrin reaction, and measurement of fluorescence. The detailed genotyping methodology was described in previous studies $(16,19)$.

Statistical analysis. Quantitative data were compared between subjects with CKD and controls using the unpaired Student's t-test. The Chi-square test was used to compare categorical data. Allele frequencies were estimated by the gene counting method. The Chi-square test was used to identify deviations from the Hardy-Weinberg equilibrium as well as genotype distributions and allele frequencies of rs6929846 of BTN2Al between subjects with CKD and controls. Multivariate logistic regression analysis was carried out with CKD as a dependent variable. Independent variables including age, gender 
Table II. Comparison of genotype distributions and allele frequencies of rs6929846 in butyrophilin, subfamily 2, member A1 gene by the Chi-square test between subjects with chronic kidney disease (CKD) and controls.

\begin{tabular}{lcccc}
\hline Genotypes & CKD $(\%)$ & Controls $(\%)$ & P-value (genotype) & P-value (allele) \\
\hline rs6929846 & & & 0.0010 & 0.0002 \\
CC & $327(75.2)$ & $1,056(82.9)$ & \\
CT & $100(23.0)$ & $208(16.3)$ & \\
TT & $8(1.8)$ & $10(0.8)$ & \\
Hardy-Weinberg P-value & 0.9112 & 0.9449 & \\
\hline
\end{tabular}

Table III. Multivariate logistic regression analysis of rs6929846 in butyrophilin, subfamily 2, member A1 gene and chronic kidney disease.

\begin{tabular}{|c|c|c|c|c|c|c|c|c|}
\hline \multirow[b]{2}{*}{ Genotype } & \multicolumn{2}{|c|}{ Dominant } & \multicolumn{2}{|c|}{ Recessive } & \multicolumn{2}{|c|}{ Additive 1} & \multicolumn{2}{|c|}{ Additive 2} \\
\hline & P-value & OR $(95 \% \mathrm{CI})$ & P-value & OR $(95 \% \mathrm{CI})$ & P-value & OR $(95 \% \mathrm{CI})$ & P-value & OR $(95 \% \mathrm{CI})$ \\
\hline rs6929846 & 0.0002 & $2.02(1.40-2.92)$ & 0.2918 & & 0.0003 & $2.00(1.38-2.91)$ & 0.2184 & \\
\hline
\end{tabular}

OR, odds ratio; CI, confidence interval. Multivariate logistic regression analysis was performed with adjustment for age, gender, body mass index, smoking status, and the prevalence of hypertension, diabetes mellitus and dyslipidemia. $\mathrm{P}<0.05$ is shown in bold.

Table IV. Association of rs6929846 in butyrophilin, subfamily 2, member A1 gene (BTN2Al) to the serum concentration of creatinine and estimated glomerular filtration rate (eGFR) in all subjects.

\begin{tabular}{lccr}
\hline & \multicolumn{2}{c}{ BTN2A1 genotype } & \\
\cline { 2 - 3 } Parameters & CC & CT + TT & P-value \\
\hline Serum creatinine $(\mu \mathrm{mol} / \mathrm{l})$ & $53.3 \pm 18.2$ & $56.9 \pm 23.6$ & 0.0107 \\
eGFR $\left(\mathrm{ml} /\right.$ min per $\left.1.73 \mathrm{~m}^{2}\right)$ & $89.3 \pm 22.3$ & $85.0 \pm 24.5$ & 0.0468 \\
\hline
\end{tabular}

(0, female; 1 , male), body mass index (BMI), smoking status ( 0 , non-smoker; 1 , current or former smoker), the prevalence of hypertension, diabetes mellitus, or dyslipidemia ( 0 , no history of these conditions; 1 , positive history), and BTN2Al genotype, as well as P-values, odds ratios, and $95 \%$ confidence intervals were also calculated. The BTN2Al genotype was assessed according to dominant, recessive, and additive genetic models. Additive models included additive 1 (heterozygotes vs. wild-type homozygotes) and additive 2 (variant homozygotes vs. wild-type homozygotes) models, which were analyzed simultaneously using a single statistical model.

$\mathrm{P}<0.05$ was considered to indicate a statistically significant difference. Statistical significance was examined by two-sided tests performed with JMP Genomics version 6.0 software (SAS Institute, Cary, NC, USA).

\section{Results}

The baseline characteristics of the subjects are shown in Table I. Age, the frequency of being male, BMI, the prevalence of hypertension, dyslipidemia, and diabetes mellitus, as well as serum concentrations of blood urea nitrogen and creatinine were higher, whereas the prevalence of smoking and eGFR was lower, in subjects with CKD than in controls.

Using the Chi-square test, a comparison of genotype distributions and allele frequencies was performed, revealing that rs6929846 of BTN2A1 was significantly $(\mathrm{P}<0.05)$ associated with CKD (Table II). The frequencies of the risk $\mathrm{T}$ allele were 0.133 and 0.089 in subjects with CKD and controls, respectively. Genotype distributions were in the Hardy-Weinberg equilibrium for subjects with CKD and controls.

Multivariate logistic regression analysis was performed with adjustment for age, gender, BMI, smoking status, and the prevalence of hypertension, diabetes mellitus, and dyslipidemia. The results demonstrated that rs6929846 of BTN2A1 was significantly associated with CKD (dominant and additive 1 models), with the minor $\mathrm{T}$ allele representing a risk factor for CKD (Table III).

The association of rs 6929846 of BTN2Al to the serum concentration of creatinine and eGFR was examined for all the individuals (Table IV). The serum concentration of creatinine was significantly higher, whereas eGFR was significantly 
lower for individuals in the combined group of CT and TT genotypes than for those with the $\mathrm{CC}$ genotype.

\section{Discussion}

Results of the present study have shown that rs6929846 of BTN2A1 was significantly associated with the prevalence of CKD in community-dwelling Japanese individuals, with the minor $\mathrm{T}$ allele representing a risk factor for this condition. Previously, we showed that rs6929846 of BTN2Al was significantly associated with CKD in a different hospital-based study population (20). The present results in the population-based study were consistent with the previous observations in the hospital-based study (20) and validate the association of rs6929846 in BTN2A1 with CKD.

The BTN2Al belongs to the BTN superfamily, and is a cell surface transmembrane glycoprotein that has roles in lipid, fatty acid, and sterol metabolism (Entrez Gene, NCBI). Although the butyrophilin family was originally identified on the basis of its ability to promote the production of milk fat globules (21), many butyrophilin and butyrophilin-like family of proteins were shown to regulate immune function, and polymorphisms in the coding sequences were related to predisposition to inflammatory diseases (22). In a previous study, we showed that the T allele of rs6929846 of BTN2Al was associated with an increased risk for myocardial infarction (16). The T allele of rs6929846 was associated with an increased transcription activity of $B T N 2 A 1$, and the serum concentrations of high sensitivity $C$-reactive protein were significantly greater in individuals in the combined group of $\mathrm{CT}$ and TT genotypes than in those with the CC genotype among healthy individuals without neoplastic, infectious, or inflammatory disease (23). These observations suggest that the T allele of rs6929846 of BTN2A1 may accelerate inflammatory processes.

Renal tubulointerstitial damage is considered a common feature in CKD (24). Findings of previous studies have shown that chronic inflammation is crucial in the promotion of interlinked fibrosis and cell injury within the tubulointerstitium, and macrophages initially mediate this inflammatory process (25). In addition, infiltrating macrophages in response to glomerular and tubular injury lead to the generation of proinflammatory cytokines (interleukin $1, \beta$; tumor necrosis factor; and transforming growth factor, $\beta$ 1), vasoactive eicosanoids, and reactive oxygen species $(26,27)$. This vicious cascade accelerates structural and functional damage, leading to the deterioration of renal function. Concerning the role of chronic inflammation in the pathogenesis of CKD, the association of rs6929846 of BTN2A1 with CKD may be attributable to acceleration of the inflammatory process by the $\mathrm{T}$ allele of this polymorphism.

There are some limitations to our study: i) given that the study subjects comprised only Japanese individuals, validation of our findings will required in other ethnic groups; ii) we used eGFR instead of a directly measured rate to define CKD. Although the equation of eGFR has not been validated in individuals $>70$ years, the best estimate of GFR for elderly Japanese individuals has not been determined; iii) we were not able to obtain information on the pathological cause of CKD in a substantial proportion of the subjects with this condition. Such information can be obtained by detailed clinical examination, including renal biopsy, however, such diagnostic procedures are not considered feasible for a genetic epidemiological study; and iv) the molecular mechanisms underlying the effects of rs6929846 of BTN2A1 on the development of CKD have not been determined.

In conclusion, the present results suggest that $B T N 2 A l$ is a susceptibility gene for CKD in community-dwelling Japanese individuals. Determination of the genotype for rs6929846 of BTN2A1 may prove informative for assessment of the genetic risk for CKD in the Japanese population.

\section{Acknowledgements}

This study was supported by a Grant-in-Aid for Scientific Research from the Ministry of Education, Culture, Sports, Science, and Technology of Japan (no. 24590746 to Y.Y.) and by Research Grants from the Japan Health Foundation and Okasan Kato Culture Promotion Foundation (to Y.Y.).

\section{References}

1. Levey AS, Eckardt KU, Tsukamoto Y, et al: Definition and classification of chronic kidney disease: a position statement from Kidney Disease: Improving Global Outcomes (KDIGO). Kidney Int 67: 2089-2100, 2005

2. Meguid El Nahas A and Bello AK: Chronic kidney disease: the global challenge. Lancet 365: 331-340, 2005.

3. Weiner DE, Tighiouart H, Amin MG, et al: Chronic kidney disease as a risk factor for cardiovascular disease and all-cause mortality: a pooled analysis of community-based studies. J Am Soc Nephrol 15: 1307-1315, 2004.

4. Couser WG, Remuzzi G, Mendis S and Tonelli M: The contribution of chronic kidney disease to the global burden of major noncommunicable diseases. Kidney Int 80: 1258-1270, 2011.

5. Kohro T, Furui Y, Mitsutake N, et al: The Japanese national health screening and intervention program aimed at preventing worsening of the metabolic syndrome. Int Heart J 49: 193-203, 2008.

6. Boulware LE, Jaar BG, Tarver-Carr ME, Brancati FL and Powe NR: Screening for proteinuria in US adults: a cost-effectiveness analysis. JAMA 290: 3101-3114, 2003.

7. Kondo M, Yamagata K, Hoshi SL, et al: Cost-effectiveness of chronic kidney disease mass screening test in Japan. Clin Exp Nephrol 16: 279-291, 2012.

8. Iseki K: The okinawa screening program. J Am Soc Nephrol 14: S127-S130, 2003

9. Haroun MK, Jaar BG, Hoffman SC, Comstock GW, Klag MJ and Coresh J: Risk factors for chronic kidney disease: a prospective study of 23,534 men and women in Washington County, Maryland. J Am Soc Nephrol 14: 2934-2941, 2003.

10. Yamagata K, Ishida K, Sairenchi T, et al: Risk factors for chronic kidney disease in a community-based population: a 10-year follow-up study. Kidney Int 71: 159-166, 2007.

11. Klag MJ, Whelton PK, Randall BL, et al: Blood pressure and end-stage renal disease in men. N Engl J Med 334: 13-18, 1996.

12. O'Seaghdha CM, Parekh RS, Hwang SJ, et al: The MYH9/APOL1 region and chronic kidney disease in European-Americans. Hum Mol Genet 20: 2450-2456, 2011.

13. Chow KM, Wong TY and Li PK: Genetics of common progressive renal disease. Kidney Int Suppl 94: S41-S45, 2005.

14. Köttgen A, Pattaro C, Böger CA, et al: New loci associated with kidney function and chronic kidney disease. Nat Genet 42: 376-384, 2010.

15. Okada Y, Sim X, Go MJ, et al: Meta-analysis identifies multiple loci associated with kidney function-related traits in East Asian populations. Nat Genet 44: 904-909, 2012.

16. Yamada Y, Nishida T, Ichihara S, et al: Association of a polymorphism of BTN2A1 with myocardial infarction and dyslipidemia in East Asian populations. Atherosclerosis 215: 145-152, 2011.

17. Matsuo S, Imai E, Horio M, et al: Revised equations for estimated GFR from serum creatinine in Japan. Am J Kidney Dis 53: 982-992, 2009. 
18. National Kidney Foundation: K/DOQI clinical practice guidelines for chronic kidney disease: evaluation, classification, and stratification. Am J Kidney Dis 39: S1-S266, 2002.

19. Itoh Y, Mizuki N, Shimada T, et al: High-throughput DNA typing of HLA-A, -B, -C, and -DRB1 loci by a PCR-SSOP-Luminex method in the Japanese population. Immunogenetics 57: 717-729, 2005.

20. Yoshida T, Kato K, Horibe H, et al: Association of a genetic variant of BTN2A1 with chronic kidney disease in Japanese individuals. Nephrology 16: 642-648, 2011.

21. Ogg SL, Weldon AK, Dobbie L, Smith AJ and Mather IH: Expression of butyrophilin (Btn1a1) in lactating mammary gland is essential for the regulated secretion of milk-lipid droplets. Proc Natl Acad Sci USA 101: 10084-10089, 2004.

22. Arnett HA, Escobar SS and Viney JL: Regulation of costimulation in the era of butyrophilins. Cytokine 46: 370-375, 2009.
23. Oguri M, Kato K, Yoshida T, et al: Association of a genetic variant of BTN2A1 with metabolic syndrome in East Asian populations. J Med Genet 48: 787-792, 2011.

24. Harris RC and Neilson EG: Toward a unified theory of renal progression. Annu Rev Med 57: 365-380, 2006.

25. Schnaper HW and Kopp JB: Why kidneys fail: report from an American Society of Nephrology Advances in Research Conference. J Am Soc Nephrol 17: 1777-1781, 2006.

26. Ricardo SD, van Goor H and Eddy AA: Macrophage diversity in renal injury and repair. J Clin Invest 118: 3522-3530, 2008.

27. Brosius FC III: New insights into the mechanisms of fibrosis and sclerosis in diabetic nephropathy. Rev Endocr Metab Disord 9: 245-254, 2008. 